Meta

Journal des tradlucteurs

Translators' Journal

\title{
Grievance : grief ou réclamation ?
}

\section{Rachel B. Quesnel et Viviane Dasilva}

Volume 15, numéro 3, septembre 1970

URI : https://id.erudit.org/iderudit/002487ar

DOI : https://doi.org/10.7202/002487ar

Aller au sommaire du numéro

Éditeur(s)

Les Presses de l'Université de Montréal

ISSN

0026-0452 (imprimé)

1492-1421 (numérique)

Découvrir la revue

Citer cet article

Quesnel, R. B. \& Dasilva, V. (1970). Grievance : grief ou réclamation? Meta, 15(3), 164-165. https://doi.org/10.7202/002487ar d'utilisation que vous pouvez consulter en ligne.

https://apropos.erudit.org/fr/usagers/politique-dutilisation/ 


\section{PROBLÈMES ET SOLUTIONS}

\section{GRIEVANCE : GRIEF OU RÉCLAMATION?}

L'étude systématique de 1a terminologie propre à une technique nous amène souvent à remettre en question le bien-fondé de termes fréquemment utilisés dans les traductions. Un récent travail sur les conventions collectives nous a mises en face du problème de l'interprétation donnée en français au mot grievance. D'abord on constate à la lecture de textes français et canadiens-français traitant de relations de travail, que le concept de grievance correspond à réclamation dans les conventions françaises et à grief dans les conventions canadiennes-françaises. $\mathrm{Y}$ a-t-il à cette disparité une explication autre qu'un simple écart de l'usage ?

Notre premier souci a été de rechercher la réalité exacte recouverte par le terme grievance. The American Heritage Dictionary of the English Language ${ }^{1}$ le définit en ces termes : a) A natural or supposed circumstance regarded as a just cause for protest; b) $A$ complaint or protestation based on such a circumstance.

En analysant cette définition, nous nous rendons compte que le terme anglais grievance décrit bien deux réalités, c'est-à-dire le concept en lui-même et l'expression de ce concept. The Random House Dictionary ${ }^{2}$ confirme cette observation.

Or dans quelle mesure peut-on considérer les mots réclamation et grief comme des équivalences de grievance?

Le mot grief est défini par Robert ${ }^{3}$ : Sujet, motif de plainte (généralement contre une personne). Ex. : Avoir des griefs contre quelqu'un; exposer, formuler ses griefs.

Le mot réclamation d'autre part y est défini : a) Action de réclamer, de

1. The American Heritage Dictionary of the English Language, William Morris, ed., New York, American Heritage Publishing Company et Houghton Mifflin, 1969.

2. The Random House Dictionary of the English Language, édition intégrale, Jess Stein et Laurence Urdang, éd., New York, Random House, 1967.

3. Le Petit Robert, Paris, Société du Nouveau Littré, 1968. 
s'adresser à une autorité pour faire reconnaître l'existence d'un droit. Ex. : Faire, déposer une réclamation; $b$ ) Protestation.

À la lumière de ces deux définitions, on constate que les deux réalités couvertes par le terme grievance exigent deux termes différents en français. En effet, un grief est une réalité vue de l'intérieur alors qu'une réclamation implique un geste extérieur, comme on le constate dans la définition même de "grief 》 où pour exprimer un mouvement vers l'extérieur, il faut utiliser une périphrase : « exposer, formuler ses griefs ».

Bénac ${ }^{4}$ apporte cette précision : grief implique simplement qu'on conçoit ou qu'on exprime en quoi on est lésé, sans y ajouter des plaintes. Et réclamer signifie protester, demander, revendiquer comme sien en justice ou autrement, ce à quoi on a un droit strict.

En feuilletant de nombreuses conventions collectives françaises et autres ouvrages sur les relations du travail, notamment les documents du Bureau international du travail, nous avons constaté que seul le terme réclamation est utilisé. Par contre, les conventions canadiennes-françaises n'utilisent que grief.

Or, il nous semble que l'utilisation de termes différents pourrait s'expliquer par une approche différente de la question. Les Français considèrent le fait sous l'aspect de la communication à établir entre le patronat et le syndicat. Dans tous ces textes, en effet, il n'est jamais question du grief « ressenti » par le salarié mais bien de la démarche entreprise pour porter ce grief à l'attention de l'intéressé.

Par contre, dans les conventions canadiennes, on utilise le mot grief selon l'acception que donnent les conventions de langue anglaise au terme grievance, c'est-à-dire qu'on se place à la fois du point de vue du salarié qui « ressent »un grief et du point de vue de la démarche de présentation de ce grief au patronat. Dans ce dernier cas, on étoffe le mot grief par une périphrase comme «présenter des griefs par écrit»ou "soumettre ses griefs». En aucun cas, on ne rencontre le terme réclamation.

Toutefois, d'autres personnes se sont déjà penchées sur ce problème de traduction. Ainsi dans Québec/Travail ${ }^{5}$ de 1967, l'auteur d'un article a soulevé la question : "Cette extension de sens que le Code du travail donne au mot grief n'est pas grave, ce mot est parfaitement acceptable et il n'y a pas de quoi fouetter un chat $»$. Il semble, toutefois, que d'autres aient repris le problème puisque le bulletin Mieux dire ${ }^{\theta}$ recommande comme traduction de Grievance Committee l'expression comité de réclamation.

Est-ce à dire qu'il serait de bon aloi, en ce cas, d'enjamber la « grande mare * pour rejoindre la subtile tournure d'esprit de nos amis français?

Lorsque la convention décrit, en particulier, la procédure d'instruction des griefs, il faut, convenir que l'usage du mot réclamation semble spécialement approprié.

Rachel B. Quesnel et Viviane Dasilva

4. Henri Bénac, Dictionnaire des synonymes, Paris, Hachette, 1956, au mot reproche, p. 817.

5. Québec/Travail, Québec, Ministère du Travail, III, 12 (décembre 1967), p. 7.

6. Mieux dire, bulletin de l'Office de la langue française, Québec, Ministère des Affaires culturelles, février 1969. 